\title{
Controle alternativo da mancha de Ramularia do algodoeiro
}

\author{
L eonardo A ngelo de A quino; Paulo Geral do Bergerr,3; Fabrício de Á vila R odrigues²; L aércio Zambolim²; Fernando \\ Ogoshi ${ }^{1}$; L ucas M. M iranda ${ }^{1} \&$ M arcelo M agri Lélis ${ }^{1}$.
}

${ }^{12}$ Departamento de Fitotecnia, UFV, CEP 36570-000, Viçosa, M G; ${ }^{12}$ Departamento de Fitopatologia, UFV, CEP 36570-000, Viçosa, M G; ${ }^{33}$ Email: pberger@ufv.br; Parte da Dissertação de M estrado do primeiro autor.

Data de chegada: 13/11/2006. A ceito para publicação em: 22/10/2007

\section{RESUMO}

A quino L .A .; B erger P.G.; RodriguesF.A .; Zambolim, L .; O goshi F.; M iranda, L.M . \& Lélis M .M . Controle alternativo da mancha de R amularia do algodoeiro. Summa Phytopathologica, v.34, n.2, p.131-136, 2008

A mancha de Ramularia, causada pelo fungo Ramularia gossypii (Speg.) Cif., é uma doença foliar de destaque na cotonicultura brasileira. Com a expansão da cotonicultura, existem poucos produtos avaliados para manejo da Ramularia, destacando-se os fungicidas dos grupos triazóis e estrobirulinas. Objetivou-se nesse trabalho avaliar 0 efeito da aplicação foliar do silicato de potássio, da cal da Viçosa e de fungicidas protetores (mancozeb e clorotalonil) para o controle químico da mancha de Ramularia. 0 silicato de potássio não foi eficiente para 0 controle da doença com severidade de $15,38 \%$, enfolhamento relativo aos 166 dias após a emergência de 38,19\%, produtividade de algodão em caroço de 136,11 @ ha-1 e área abaixo da curva de progresso da mancha de Ramularia de 644, 598 e 172 nos terços inferior, médio e superior, respectivamente, com valores semelhantes à testemunha sem fungicida. A calda viçosa proporcionou satisfatório controle da doença não diferindo em termos de produtividade dos tratamentos com piraclostrobin e ou tebuconazol, sendo o incremento de produtividade em relação à testemunha de $88 \%$. Os fungicidas mancozeb e clorotalonil em mistura com calda Viçosa ou com tebuconazole foram eficientes no controle da mancha de Ramularia, destacando-se o fungicida mancozeb em mistura com tebuconazol.

Palavras-chave: nutrição de plantas, silício, Ramularia gossypii.

\section{ABSTRACT}

A quino L.A .; B erger P.G.; Rodrigues F.A .; Zambolim, L.; O goshi F.; M iranda, L.M . \& L élis M .M. A Iternative control of the Cotton ramularia spot. Summa Phytopathologica, v.34, n.2, p.131-136, 2008

A reolate mildew, caused by the fungus Ramularia gossypii (Speg.) Cif., is the most important foliar disease on cotton. There are a few products available to the growers to manage this disease among them the fungicides tryazol and strobilurine. This study aimed to evaluate the effect of foliar application of potassium silicate, 'calda Viçosa' e protector fungicides (M ancozeb and Clorothalonyl) to control areolate mildew on cotton. The potassium silicate was not efficient to control the disease with severity of $15.38 \%$, canopy quality at 166 days after plant emergence of $38.19 \%$, yield of $2041.65 \mathrm{~kg} \mathrm{ha}^{-1}$ and area under areolate mildew progress curve of 644,598 and 172 at the lower, medium and higher canoy, respectively, with these all values being similar to the check treatment (no fungicide applied). The treatment with 'Calda Viçosa' gave the best disease control and did not differ from the treatments with Pyraclostrobin or Tebuconazole in terms of yield, but showed an increase of $88 \%$ in yield compared to the check treatment. The fungicides $M$ ancozeb and Clorothalonyl mixed with 'Calda Viçosa' or with Tebuconazole were efficient to control the disease, especially the mixture of Mancozeb with Tebuconazole.

Additional keywords: plant nutrition, silicon, Ramularia gossypii.

No B rasil, devido às condições climáticas favoráveis, são crescentes os problemas com doenças em al godoeiro, como, por exemplo, a mancha de Ramularia [Ramularia gossypii (Speg.) Cif. forma anamórfica ou M ycosphaerella areola (J . Ehrlich \& F. A. Wolf) forma teleomórfica], também conhecida como falso oídio $(6,16)$. Esta doença encontra-se disseminada em praticamente todas as áreas de cultivo do país (2). Outrora era tida como de importância secundária no al godoeiro, pois ocorria no final do ciclo da cultura e não comprometia a produção. A tualmente, no entanto, tem merecido destaque em função de sua ocorrência desde o início do ciclo da cultura, promovendo desfolha precoce, o que acarreta em redução da produção e da qualidade da fibra, além do custo de manejo $(10,19)$. Cia et al. (6) demonstraram que nas condições edafoclimáticas do $M$ ato Grosso, a redução de produtividade pode chegar a $75 \%$ em variedades mais suscetíveis à doença.

Os sintomas consistem de lesões angulosas entre as nervuras, medindo de 1 a $3 \mathrm{~mm}$ inicialmente, de coloração branca e, posteriormente, amarelada de aspecto pulverulento, caracterizado pela 
esporulação do patógeno (2). No caso de intensa severidade, as manchas agregam-se e provocam a queda das folhas. Em períodos chuvosos, podem ocorrer sintomas precoces, chegando a provocar queda de folhas e apodrecimento das maçãs dos ramos mais próximos do solo (19).

0 manejo desta doença tem sido feito por meio da integração de medidas de manejo, como o uso de cultivares com resistência parcial, época de plantio e principal mente aplicação de fungicidas $(5,21)$. Todavia, a principal estratégia de manejo adotada atualmente é o uso do controle químico $(5,15,19)$. 0 controle químico é apresentado como al ternativa para garantir o desenvolvimento da cultura na região dos Cerrados, visto que a maioria dos cultivares de algodão utilizados não possui resistência vertical à doença (5). 0 controle químico tem sido de modo geral eficiente, no entanto, este implica num custo a mais na lavoura, o qual é em média de U\$ 40,00 ha $^{-1}$ por aplicação, sendo necessárias de três a quatro aplicações durante o ciclo da cultura. Dentre os fungicidas para controle químico destacam-se os triazóis e as estrobirulinas $(5,15$, 19).

$\mathrm{N}$ a década de 70 , com o surgimento da ferrugem do cafeeiro ( $\mathrm{H}$ emileia vastatrix B erk. Et B r.), pesquisas foram desenvolvidas no departamento de Fitopatologia da Universidade Federal de Viçosa no intuito de avaliar e desenvolver produtos para auxiliar no manejo da doença. Para tal, foi desenvolvida a calda Viçosa, uma suspensão coloidal, composta de fertilizantes complexados com cal hidratada, a qual além do efeito sobre a doença, funciona também como fertilizante foliar (7). A cal da é composta por uma mistura de sulfatos de cobre, zinco, magnésio e ácido bórico, mais cal hidratada para ajustar o pH para 7 a 7,5. A pós o uso inicial em café, novas pesqui sas foram real izadas para a sua utilização em outras cul turas, como tomate (25) e feijão (9), mostrando-se eficiente no controle de doenças da parte aérea. A utilização da calda Viçosa no manejo de doenças é uma opção de baixo custo, eficiente e não agride 0 meio ambiente, que além do controlar doenças, fornece micronutrientes como cobre, boro e zinco.

O silício (Si), embora não atenda os critérios de essencialidade de um nutriente, desempenha importantes papéis na planta como redução do acamamento e aumento da resistência a doenças. Em gramíneas e em al gumas dicotiledôneas como pepineiro e tomateiro, têm-se verificado redução da severidade de doenças com a aplicação de Si, no solo ou em solução nutritiva (8). Em arroz, importantes doenças fúngicas como a brusone e a mancha parda tiveram suas intensidades significativamente reduzidas com a aplicação de Si no solo (8). A literatura também registra o efeito positivo do Si no controle de doenças fúngicas em dicotiledôneas cultivadas comercialmente, como o do míldio pulverulento do pepino, abóbora e melão (18), de Pythium ul timum e murcha-de-Fusário em pepino (F usarium oxysporum f.sp. cucumerinum) (3). 0 efeito do Si sobre a redução de doenças é explicado pela silificação das células epidérmicas, o que reduziria a penetração dos conídios (13). De acordo com Chérif et al. (4), pode haver a produção de compostos fenólicos com ação fungitóxica. Estudos recentes têm sugerido que além do ef eito mecânico sobre a penetração dos esporos do patógeno no hospedeiro (13), o Si está envolvido com o acúmulo de compostos antifúngicos, ativação de enzimas relacionadas com a defesa e fitoalexinas $(4 ; 22,23)$.

Objetivou-se nesse trabal ho avaliar o efeito da calda Viçosa e de fungicidas protetores, mesostêmicos e sistêmicos, e do silicato de potássio aplicado via foliar, no progresso da mancha de R amularia do algodoeiro.

\section{MATERIAL E MÉTODOS}

\section{Condições de cultivo}

o experimento foi conduzido em condições campo em sistema de plantio direto, na U niversidade Federal de Viçosa, no período de novembro de 2005 a junho de 2006, sob ocorrência natural da mancha de R amularia. U tilizou-se a cultivar de algodão ' $D$ el ta 0 pal' em razão da sua suscetibilidade à doença e pelas características agronômicas e comerciais desejáveis. 0 solo da área experimental é classificado como A rgissolo Vermelho A marelo, textura argilosa, com as seguintes características químicas: $\mathrm{pH}$ em $\mathrm{H}_{2} \mathrm{O}$ (relação $1: 2,5$ ) $=5,4 ; \mathrm{P}=27,7$ $\mathrm{mg} \mathrm{dm}^{-3} ; \mathrm{K}=130 \mathrm{mg} \mathrm{dm}^{-3}$ (P-K : extrator $\mathrm{M}$ ehlich 1$) ; \mathrm{Ca}^{2+}=38 \mathrm{mmol}_{\text {。 }}$ $\mathrm{dm}^{-3} ; \mathrm{M} \mathrm{g}^{2+}=6 \mathrm{mmol}_{\mathrm{c}} \mathrm{dm}^{-3}\left(\mathrm{C}\right.$ a e $\mathrm{M} \mathrm{g}$ : extrator $\left.\mathrm{KCl} 1 \mathrm{~mol} \mathrm{~L}^{-1}\right) ; \mathrm{H}+\mathrm{Al}=$ $47,9 \mathrm{mmol}_{\mathrm{c}} \mathrm{dm}^{-3}$ (extrator acetato de cál cio $0,5 \mathrm{~mol} \mathrm{~L}^{-1}$ a pH 7,0); SB $=47,3 \mathrm{mmol}_{\mathrm{cm}} \mathrm{dm}^{-3} \mathrm{~V}=50 \% ; \mathrm{m}=0 \% ; \mathrm{M} .0=32 \mathrm{~g} \mathrm{~kg}^{-1} \mathrm{e}$ - -rem $=23,8$. A adubação de plantio constou de $250 \mathrm{~kg} \mathrm{ha}^{-1}$ do formulado 8-28-16.

Tabela 1. Relação dos tratamentos usados no experimento.

\begin{tabular}{|c|c|}
\hline Tratamentos & Doses* \\
\hline 1. Sem fungicida (Testemunha) & -- \\
\hline 2. Silicato de potássio - SP $1^{1 /}$ & 3,6 \\
\hline 4. Calda Viçosa (Viça Café ${ }^{\circledast}$ comum $^{2}$ ) alternado com SP2 - CV ou SP2/1 & 5,0 e 4,8 \\
\hline 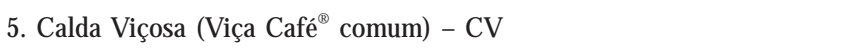 & 5,0 \\
\hline 6. Calda Viçosa (Viça Café ${ }^{\circledast}+$ Silicato de potássio**)- CV +SP & 5,0 \\
\hline 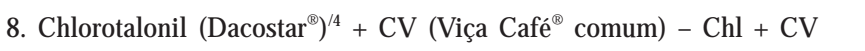 & $2,0+2,0$ \\
\hline 9. $M$ ancozeb $\left(\right.$ M anzate $\left.{ }^{\circledast}\right)+$ Triazol $\left(\right.$ Folicur $\left.^{\circledast}\right)-T R+M Z$ & $3+0,3$ \\
\hline 10. Chlorotalonil $\left(\right.$ Dacostar $\left.{ }^{\circledR}\right)+$ Triazol $\left(\right.$ Folicur $\left.^{\circledR}\right)-T R+C h l$ & $2+0,3$ \\
\hline 11. Tebuconazole (Folicur ${ }^{\circledR / 5}$ ) - TR & 0,6 \\
\hline 12. Piraclostrobin $\left(\right.$ Comet $\left.^{\circledR / 6}\right)$ - EST & 0,4 \\
\hline
\end{tabular}

* Kg ou L do produto comercial ha ${ }^{-1}$;* Silicato de potássio comercializado juntamente com o Viça Café $e^{\oplus} ;{ }^{11}$ Produto comercial FertiSil ${ }^{\circledR}$ (INEOS Silicas Brasil); ${ }^{12} \mathrm{~K}=10, \mathrm{~S}=10, \mathrm{Cu}=10, \mathrm{Zn}=6, \mathrm{~B}=3, \mathrm{Mn}=2 \mathrm{e} \mathrm{M} \mathrm{g}=1 \% ;{ }^{13} 800 \mathrm{~g} \mathrm{~kg}^{-1}$ de Mancozeb (formulação pó molhável); ${ }^{14} 750 \mathrm{~g} \mathrm{~kg}^{-1}$ de Clorotalonil (formulação pó molhável); ${ }^{15} 200 \mathrm{~g} \mathrm{~L}^{-1}$ de Tebuconazole (formulação concentrado emulsionável); ${ }^{16} 250 \mathrm{~g} \mathrm{~L}^{-1}$ de Piraclostrobin (formulação concentrado emulsionável); CV = calda Viçosa. 
Em cobertura, aplicou-se $70 \mathrm{~kg} \mathrm{ha}^{-1}$ de $\mathrm{N}$ na forma de sulfato de amônio parcelados em duas aplicações aos 40 e 60 dias após a emergência (DA E). D urante a condução do experimento, real izaramse aplicações do herbicida paraquat (Gramoxone ${ }^{\circledR}$ - 2 I ha-1) em jato dirigido para o controle de plantas daninhas. Foram realizadas aplicações de inseticidas $\left(\operatorname{Tracer}^{\circledR}\right.$, Endosulfan $^{\circledR}{ }^{\circledR}$ Decis ${ }^{\circledR}$ ) nas dosagens recomendadas para a cultura para controle de insetos-pragas. As irrigações foram realizadas por aspersão convencional.

\section{Tratamentos}

Os tratamentos de 1 a 13, constituíram-se de produtos para 0 manejo da Ramularia, isolados ou em misturas, além da testemunha (sem fungicida) (Tabela 01). A unidade experimental constitui-se de três linhas espaçadas de $0,9 \mathrm{~m}$ com $10 \mathrm{~m}$ de comprimento, sendo considerada como útil a linha central, excetuando-se $1 \mathrm{~m}$ de cada extremidade. Utilizou-se o delineamento experimental em blocos casualizados, com quatro repetições.

Foram realizadas cinco aplicações dos tratamentos de 2 a 8 com intervalo de 21 dias (produtos protetores) e três aplicações dos tratamentos de 9 a 13 a intervalo de 30 dias (produtos sistêmicos ou sistêmicos em mistura com protetores) (Tabela 1). Iniciaram-se as aplicações quando foram observados os primeiros sinais da doença, 0

Tabela 2. Á rea abaixo da curva de progresso da mancha de ramularia (A ACPMR) do algodoeiro e taxa aparente de infecção dos 54 aos 110 DAE ( $r_{54-110}$ ) e dos 117 aos 145 DAE $\left(r_{117-145}\right)$ nos terços inferior (TI), médio (TM) e superior (TS) nos tratamentos para 0 controle da doença.

\begin{tabular}{|c|c|c|c|c|c|c|c|c|c|}
\hline \multirow[t]{2}{*}{ Tratamentos } & \multicolumn{3}{|c|}{ AACPMR } & \multicolumn{3}{|c|}{$r_{54-110}$} & \multicolumn{3}{|c|}{$r_{117-145}$} \\
\hline & $\mathrm{TI}$ & TM & TS & $\mathrm{TI}$ & TM & TS & $\mathrm{TI}$ & TM & TS \\
\hline 1. Testemunha & $886,28 a^{11}$ & 709,57 a & $229,49 a$ & 0,2278 a & $0,2406 a$ & 0,0413 a & $0,9855 \mathrm{a}$ & 0,9171 a & $0,4538 a$ \\
\hline 2. Silicato de potássio - SP1/2 & $593,00 \mathrm{~b}$ & 585,86 a & $162,23 \mathrm{a}$ & 0,1515 a & $0,0859 \mathrm{~b}$ & $0,0119 \mathrm{~b}$ & $1,0841 \mathrm{a}$ & 1,0008 a & $0,3554 a$ \\
\hline 3. Silicato de potássio - SP2/3 & $644,73 \mathrm{~b}$ & 598,71 a & $172,21 \mathrm{a}$ & $0,0919 \mathrm{~b}$ & $0,1062 b$ & $0,0043 b$ & $1,2547 \mathrm{a}$ & $1,0887 \mathrm{a}$ & $0,4422 a$ \\
\hline 4. Calda Viçosa alternado SP2 & $503,89 \mathrm{~b}$ & $421,58 \mathrm{~b}$ & $118,33 \mathrm{~b}$ & $0,0847 \mathrm{~b}$ & $0,0901 \mathrm{~b}$ & $0,0118 \mathrm{~b}$ & 0,9394 a & 0,8560 a & $0,4942 a$ \\
\hline 5. Calda Viçosa & $298,54 \mathrm{C}$ & $183,40 \mathrm{c}$ & $32,60 \mathrm{c}$ & $0,0291 \mathrm{~b}$ & $0,0208 \mathrm{c}$ & $0,0017 \mathrm{~b}$ & $0,7472 \mathrm{~b}$ & $0,5988 \mathrm{~b}$ & $0,1759 \mathrm{~b}$ \\
\hline 7. Calda Viçosa + M ancozeb & $183,63 \mathrm{c}$ & $137,64 \mathrm{c}$ & $24,74 \mathrm{c}$ & $0,0343 b$ & $0,0426 \mathrm{c}$ & $0,0021 \mathrm{~b}$ & $0,2163 \mathrm{c}$ & $0,2115 \mathrm{c}$ & $0,0562 \mathrm{~b}$ \\
\hline 8. Calda Viçosa + Clorotalonil & $232,82 \mathrm{c}$ & $150,36 \mathrm{c}$ & $29,80 \mathrm{c}$ & $0,0227 \mathrm{~b}$ & $0,0157 \mathrm{c}$ & $0,0032 \mathrm{~b}$ & $0,3067 \mathrm{c}$ & $0,2369 c$ & $0,0985 \mathrm{~b}$ \\
\hline 9. Tebuconazole + M ancozeb & $146,29 d$ & $131,02 \mathrm{c}$ & $28,80 \mathrm{c}$ & $0,0198 \mathrm{~b}$ & $0,0220 \mathrm{c}$ & $0,0056 \mathrm{~b}$ & $0,3185 \mathrm{c}$ & $0,2883 \mathrm{c}$ & $0,0969 \mathrm{~b}$ \\
\hline 10. Tebuconazole + Clorotalonil & $349,62 \mathrm{c}$ & $285,05 \mathrm{c}$ & $92,23 \mathrm{~b}$ & $0,0221 \mathrm{~b}$ & $0,0266 \mathrm{c}$ & $0,0082 \mathrm{~b}$ & $0,6118 b$ & $0,5355 \mathrm{~b}$ & $0,2472 \mathrm{~b}$ \\
\hline 11. Tebuconazole & $11,91 \mathrm{~d}$ & $7,29 \mathrm{c}$ & $2,60 \mathrm{c}$ & $0,0372 \mathrm{~b}$ & $0,0360 \mathrm{c}$ & $0,0085 \mathrm{~b}$ & $0,1368 d$ & $0,0433 \mathrm{c}$ & $0,2217 \mathrm{~b}$ \\
\hline
\end{tabular}

${ }^{11}$ Grupos de médias seguidas pela mesma letra em cada coluna, não diferem entre si pelo critério Scott $\mathrm{K}$ nott ao nível de $5 \%$ de probabilidade; ${ }^{12} \mathrm{SP}=$ silicato de potássio; ${ }^{12,13}$ Cinco aplicações de 3,6 e $4,8 \mathrm{~kg} \mathrm{ha}^{-1}$ de silicato de potássio, respectivamente.

Tabela 3. Severidade média da mancha de ramularia do algodoeiro nos terços inferior (TI), médio (TM) e superior (TS) aos 110 (Y ${ }_{\text {máx110 }}$ ) e 145 dias após a emergência $\left(Y_{\text {máx145 }}\right)$ nos tratamentos para 0 controle da doença.

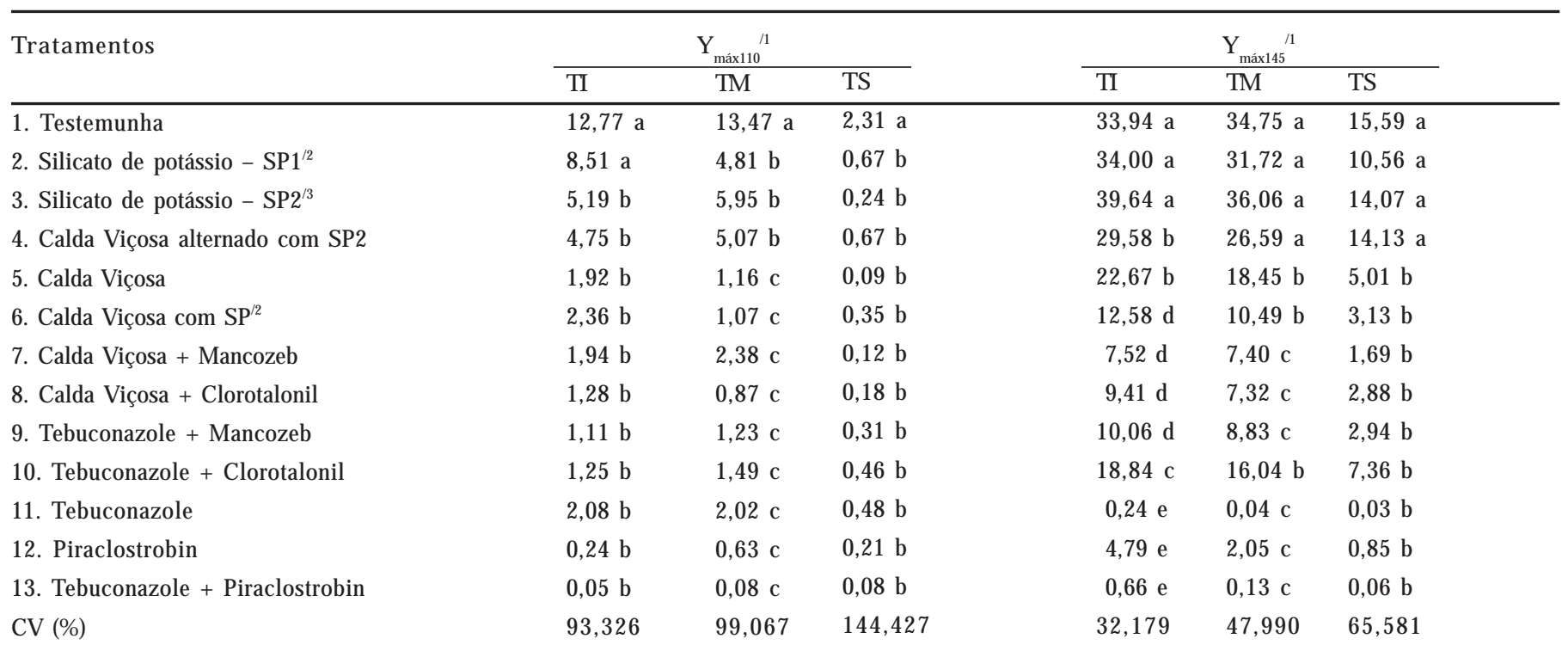

1 Severidade na nona e décima quarta semanas de avaliação, respectivamente; ${ }^{12}$ Grupos de médias seguidas pela mesma letra em cada coluna, não diferem entre si pelo critério de Scott K nott ao nível de $5 \%$ de probabilidade; ${ }^{12,13}$ Cinco aplicações de 3,6 e 4,8 $\mathrm{kg}^{-1}$ ha de silicato de potássio, respectivamente. 
que ocorreu aos 54 DAE. Utilizou-se volume de calda de $200 \mathrm{~L}$ ha-1, sendo os produtos aplicados com pulverizador costal manual equipado com bico XR 8003 (TEEJET) com regulador de pressão de $28 \mathrm{Ib} \mathrm{pol}^{-}$ 2, até atingir o ponto de escorrimento. 0 teor de potássio nos tratamentos Testemunha, SP1 e SP2 foram padronizados utilizando solução de cloreto de potássio $\left(61,728 \mathrm{~g} \mathrm{~L}^{-1}\right) \mathrm{com} \mathrm{pH}$ 5,5, equilibrado com solução diluída de $\mathrm{HCl}\left(1 \mathrm{~mol} \mathrm{~L}^{-1}\right)$. A ssim, Testemunha e SP1 receberam via foliar a mesma quantidade de K que o tratamento SP2.

Avaliações da mancha de R amularia e produtividade de algodão em caroço

Semanal mente, a partir da primeira aplicação dos tratamentos para o manejo da mancha de Ramularia, avaliou-se a severidade média nos terços inferior, médio e superior, por quatorze semanas, de acordo com a escala descrita por A quino (1). Considerou-se como terço inferior às fol has dispostas até 070 nó do ramo principal (haste), terço médio do 8-14ํnó e terço superior acima do 15ํnó. A o 166 D A E col etaramse duas plantas em cada parcela, representativas de cada tratamento, para se determinar individual mente a severidade e a área de cada folha. A área foliar foi medida utilizando-se o integrador de área foliar (Licor A rea $M$ eter 3100). Com a área foliar e a severidade da doença em cada folha, obteve a severidade média de cada planta bem como 0 enfol hamento relativo em cada tratamento, considerando o tratamento com maior enfol hamento como 100\%. A os 211 DAE, realizou-se a colheita determinando-se a produtividade de algodão em caroço e a massa média de capulhos.

A os 131 DAE, coletaram-se folhas do ramo principal no terço médio, as quais foram lavadas com água destilada e colocadas para secar em estufa com ventilação forçada de ar a $70^{\circ} \mathrm{C}$, até atingir peso constante, para determinação do teor de Si (14).

\section{A nálise estatística dos dados}

Calculou-se a taxa aparente de progresso da mancha de Ramularia (r) dos 54 aos 110 DAE $\left(r_{54-110}\right)$ e dos 117 aos 145 DAE $\left(r_{117-145}\right)$ com base nos dois picos da doença observados na testemunha aos $110 \mathrm{e}$ $145 \mathrm{DAE}$, respectivamente. Compararam-se as severidades das plantas de cada tratamentos nos picos da doença na testemunha $\left(Y_{\text {máx110 }} \mathrm{eY} Y_{\text {máx145 }}\right)$, correspondentes a nona e décima quarta semanas de avaliação, respectivamente. Com os dados da severidade da doença em cada terço, calculou-se a área abaixo da curva de progresso da mancha de ramularia (A A CPM R) (24). O s dados das características avaliadas foram submetidos à análise de variância utilizando-se o programa estatístico SA EG (UFV), sendo as médias dos tratamentos comparadas, pelo critério de S cott $\mathrm{K}$ nott ao nível de $5 \%$ de probabilidade.

\section{RESULTADOS E DISCUSSÃO}

Os tratamentos com silicato de potássio (SP1 eSP2) e sem fungicida (testemunha) diferiram quanto a A A CPM R apenas no terço inferior (Tabela 2). A taxa aparente de infecção da mancha de Ramularia $\left(r_{54 .}\right.$

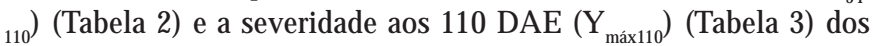
tratamentos com silicato de potássio diferiu da testemunha, exceto para o tratamento SP1 no terço inferior. É provável que a testemunha não diferiu dos tratamentos SP1 e SP2 em relação a A ACPM R nos terços médio e superior devido a maior infecção pelo patógeno nessas porções da planta em função do aumento de inóculo ocorrido no terço inferior associado às condições de microclima favoráveis ao progresso da doença. R esultados semelhantes foram obtidos entre a testemunha e os tratamentos com silicato de potássio (SP1 e SP2) em relação à taxa aparente de infecção no segundo pico da doença $\left(r_{117-145}\right)$. Segundo Paiva (19), o fechamento do dossel das plantas proporciona um microclima favorável para o progresso da doença, justificando assim as maiores taxas aparente de infecção dos 117 aos 145 DAE no experimento. Não houve diferença significativa entre os tratamentos com silicato de potássio e testemunha quanto aos teores foliares de Si na massa seca, sendo 0 teor de Si na testemunha, SP1, SP2 e no tratamento em que se alternou a aplicação do silicato de potássio com a calda Viçosa de 0,$51 ; 0,53$; 0,49 e 0,51 dag de $\mathrm{Si} \mathrm{kg}^{-1}$, respectivamente. A produtividade de al godão em caroço e a massa média de capul ho dos tratamentos com silicato de potássio e testemunha não diferiram entre si ressaltando que a

Tabela 4. Enfolhamento relativo e severidade da mancha de Ramularia do algodoeiro aos 166 dias após a emergência, massa média de capulho (M M C) e produtividade de algodão em caroço em função dos tratamentos para o controle da doença.

\begin{tabular}{|c|c|c|c|c|}
\hline Tratamentos & Enfolhamento relativo ${ }^{11}$ & Severidade (\%) & M M C (g) & Produtividade(@ ha ${ }^{-1}$ ) \\
\hline 1. Testemunha & $21,39 \mathrm{~b}^{14}$ & $16,25 a$ & $4,58 \mathrm{~b}$ & $129,63 \mathrm{~b}$ \\
\hline 2. Silicato de potássio - SP1/2 & $33,50 \mathrm{~b}$ & 15,38 a & $4,16 \mathrm{~b}$ & $129,39 \mathrm{~b}$ \\
\hline 4. Calda Viçosa alternado com SP2 & $31,10 \mathrm{~b}$ & $9,36 \mathrm{~b}$ & $4,17 \mathrm{~b}$ & $147,45 \mathrm{~b}$ \\
\hline 5. Calda Viçosa & 63,76 a & $3,94 \mathrm{c}$ & 5,06 a & 239,35 a \\
\hline 6. Calda Viçosa com SP/2 & 56,46 a & $3,74 \mathrm{c}$ & $4,70 \mathrm{a}$ & 249,53 a \\
\hline 8. Calda Viçosa + Clorotalonil & 71,89 a & $4,43 \mathrm{c}$ & $4,55 \mathrm{~b}$ & 209,25 a \\
\hline 9. Tebuconazole + M ancozeb & 58,06 a & $4,72 \mathrm{c}$ & 5,14 a & 207,64 a \\
\hline 10. Tebuconazole + Clorotalonil & $52,72 b$ & $5,59 \mathrm{c}$ & 4,79 a & 185,88 a \\
\hline 11. Tebuconazole & 94,12 a & $0,76 \mathrm{c}$ & $4,84 \mathrm{a}$ & 233,33 a \\
\hline 12. Piraclostrobin & 70,83 a & $2,25 \mathrm{c}$ & $4,84 \mathrm{a}$ & 243,98 a \\
\hline
\end{tabular}

${ }^{11}$ Enfolhamento relativo = área foliar do tratamento / área foliar final do tratamento Tebuconazole mais Piraclostrobin (maior área foliar final; $0,57 \mathrm{~m}^{2}$ planta $\left.{ }^{-1}\right) ;{ }^{14}$ Grupos de médias seguidas pela mesma letra, em cada coluna, não diferem entre si pelo critério de Scott $\mathrm{K}$ nott ao nível de $5 \%$ de probabilidade; 12,13 Cinco aplicações de 3,6 e 4,8 $\mathrm{kg} \mathrm{ha}^{-1}$ de silicato de potássio, respectivamente. 
produtividade das plantas nesses tratamentos foi, em média, $46 \%$ menor do que a produtividade obtida com os tratamentos estrobirulina e triazol (Tabela 4).

Os tratamentos com estrobirulina (Piraclostrobin) e triazol (Tebuconazole) isolados ou em mistura, foram os que apresentaram menores valores de A A CPM R, taxa aparente de infecção da R amularia $\left(r_{54-110}\right.$ e $\left.r_{117-145}\right)$, severidade $\left(Y_{\text {máx110 }}\right.$ e $\left.Y_{\text {máx145 }}\right)$ e severidade aos 166 DAE (Tabelas 2, 3 e 4). Fungicidas do grupo das estrobirulinas e triazol também foram eficientes no controle da mancha de R amularia e proporcionaram aumento de produtividade em outros trabalhos (5, 12). 0 acréscimo em produtividade com relação à testemunha, promovido pela aplicação de estrobirulina e ou triazol foi, em média, de $88 \%$.

A calda V içosa (Viça C afé ${ }^{\circledR}$ ) controlou de forma eficiente a mancha de R amularia principalmente até aos $110 \mathrm{DA} E$, mantendo a severidade em torno de 2,36; 1,16 e 0,35\% (maiores valores dos tratamentos com calda Viçosa) nos terços inferior, médio e superior, respectivamente (Tabela 3). Com relação à A A C PM R, os tratamentos com calda V içosa isolada ou em mistura com clorotalonil ou mancozeb tiveram comportamento intermediário entre a testemunha (sem fungicida) e os melhores tratamentos (triazol e estrobirulina) no terço inferior. Nos terços médio e superior, a A A CPM R nos tratamentos com cal da Viçosa não diferiram dos tratamentos com triazol ou estrobirulina (Tabela 2). Não houve diferença entre os tratamentos com calda Viçosa, isolada ou em mistura e os tratamentos com estrobirulina e ou triazol quanto à taxa aparente de infecção da mancha de R amularia no primeiro pico da doença $\left(r_{54-110}\right)$. No segundo pico de doença, a calda Viçosa isolada apresentou maior valor de taxa aparente de infecção da Ramularia $\left(r_{117}\right.$. ${ }_{145}$ ) nos terços inferior e médio, comparativamente aos tratamentos da calda viçosa em mistura com silicato, mancozeb e clorotalonil. Os valores de severidade considerando toda a planta aos 166 DAE e 0 enfolhamento relativo nos tratamentos com cal da viçosa, não diferiram dos tratamentos com triazol e estrobirulina (Tabela 4). A mistura da cal da Viçosa com os fungicidas mancozeb ou clorotalonil propiciou menores valores de severidade nos terços inferior e médio aos 145 DA E em relação a cal da V içosa isolada (Tabela 3). N o entanto, apesar da diferença na severi dade da mancha de Ramularia, não houve diferença na produtividade de algodão em caroço dos tratamentos com calda Viçosa isolada ou em mistura. N os tratamentos em que a cal da V içosa ou calda Viçosa com silicato de potássio foi aplicada isoladamente, utilizou-se uma dose 2,5 vezes maior que no tratamento em mistura com mancozeb ou clorotal onil. A maior dose da cal da Viçosa pode ter beneficiado 0 al godoeiro em termos de suprimento de micronutrientes, compensando a maior severidade da ramularia em relação aos tratamentos com cal da V içosa associados ao mancozeb ou clorotal onil. No tratamento em que se alternou as aplicações de calda Viçosa com silicato de potássio, os val ores de severidade, A A CPM R e taxa aparente de infecção da mancha de Ramularia no segundo pico $\left(r_{117-145}\right)$ foram similares aos tratamentos com silicato de potássio e testemunha (Tabelas 2, 3 e 4). Como o silicato de potássio não mostrou-se eficiente no controle da doença, pode-se afirmar que a cal da Viçosa aplicada a intervalo de 42 dias (al ternância com silicato de potássio) foi ineficiente no controle da mancha de R amularia. A calda Viçosa funciona como um fungicida protetor devido ao ef eito do cobre, sendo necessário cobrir as fol has novas a fim de se formar uma camada protetora. Tal camada permite a manutenção de certa concentração de cobre na água, que afeta a germinação dos conídios do fungo. A produtividade de al godão em caroço e a massa média de capulho nos tratamentos com calda Viçosa isolada ou em mistura com mancozeb ou clorotalonil não diferiram dos tratamentos com estrobirulina e ou triazol, exceto a massa média de capul ho do tratamento clorotal onil com cal da Viçosa. Em al godão, não existem relatos do uso da cal da Viçosa no manejo de doenças foliares. Em outras culturas como café, tomate e feijão vários autores relataram efeito positivo no controle de doenças promovido pela calda V içosa, comparativamente a fungi cidas comumente utilizados nessas culturas para manejo de doenças da parte aérea $(9,11,25)$.

0 fungicida mancozeb promoveu melhor controle da mancha de R amularia comparativamente ao clorotal onil quando em mistura com triazol (Tabelas 2, 3 e 4). Tal fato pode ser devido a maior fungitoxicidade e ou tenacidade do mancozeb em relação ao clorotal onil. Possivel mente houve um efeito aditivo maior do triazol com o mancozeb em relação à mistura com cl orotal onil, pois quando em mistura com a cal da Viçosa, os fungicidas mancozeb e clorotal onil não diferiram entre si no controle da Ramularia e no aumento da produtividade.

Calda Viçosa, mancozeb e clorotalonil (em mistura com cal da Viçosa ou triazol) mostraram-se eficientes no controle da mancha de Ramularia. 0 uso desses produtos em detrimento do uso exclusivo dos triazóis e estrobirulinas é interessante pela redução do custo de controle da doença, menor probabilidade de selecionar populações resistentes do patógeno, pelo modo de ação não específico e, adicionalmente suprir micronutrientes como B, Zn e $\mathrm{Cu}$ ao algodoeiro por meio da cal da Viçosa.

\section{AGRADECIMENTOS}

A o CN Pq pela bolsa de M estrado concedida ao primeiro autor. A empresa D elta and Pine L and Company pela gentileza em fornecer as sementes de al godão utilizadas nos experimentos. A empresa INEOS Silicas Brasil por fornecer o produto FertiSil ${ }^{\circledR}$. A FAPEM IG pelo recurso financeiro. A todos os colaboradores que di reta ou indiretamente possibilitaram a realização desse trabal ho.

\section{REFERÊNCIASBIBLIOGRÁFICAS}

1. A quino, L.A.; Escala diagramática e controle alternativo da da mancha de ramulária do algodoeiro. 44f. Dissertação (M estrado em Fitotecnia) - Universidade Federal de Viçosa, Viçosa.

2. Cassetari Neto, D.; M achado, R.S.S.; Faria, A.Y.K. Comportamento de genótipos de algodão em relação às doenças fúngicas no M ato Grosso. Fitopatologia Brasileira, Brasília, v.25, p. 362363, 2000. Suplemento.

3. Chérif, M.\& Bélanger, R. R. U se of potassium silicate amendments in recirculating nutrient solutions to suppress Phytium ultimum on long English cucumber. Plant Disease, v. 76, p. 1008-1011, 1992.

4. Chérif, M.; Benhamou, N.; Bélanger, R.R. Ultrastructural and cytochemical studies of fungal development and host reactions in cucumber plants infected by Pythium ultimum. Physiology and Molecular Plant Pathology, v. 31, p. 353-375, 1992.

5. Chitarra, L.G.; M eira, S.A.; M enezes, V.L. Controle químico da mancha de Ramulária do algodoeiro, causada por Ramularia areola, em função da idade da planta e severidade da doença - Safra 2003/2004. Campina Grande: EM BRA PA ALGODÃ O, 2005. 16 p. (Boletim de Pesquisa e Desenvolvimento, 58).

6. Cia, E.; Fuzatto, M.G.; Chiavegato, E.J.; Farias, F.J.C.; A raújo, A.E. Desempenho de cultivares e linhagem de algodoeiro diante da incidência de Ramularia. In: II CONGRESSO BRASILEIRO DE ALGODÃO, 1999, Ribeirão Preto. Anais. Campina Grande: EM BRAPA-CNPA, 1999. p. 468-470.

7. Cruz Filho, J.; Chaves, G.M. Calda Viçosa no controle da ferrugem do cafeeiro. Viçosa, MG: UFV, 1989. 22 p. (Informe Técnico, 51). 
8. Datnoff, L. E.; Deren, C. W.; Snyder, G. H. Silicon fertilization for diseases management of rice in Florida. Crop Protection, $v$. 16, p. 525-531, 1997.

9. Ferreira, G.S. Efeito da calda Viçosa na nutrição do feijoeiro e no controle da mancha-angular. 1998. 49 f. Dissertação (Mestrado em Fitotecnia) - Universidade Federal de Viçosa, Viçosa.

10. FUNDAÇÃO MT. Boletim de pesquisa de algodão, Rondonópolis, M T: Fundação M T, 2001. 238 p. (Fundação M T. Boletim, 04).

11. Herrera, R.A.U. Controle da ferrugem, da cercosporiose e do bicho mineiro e nutrição do cafeeiro com aplicação da calda Viçosa. 1994. 77 f. Dissertação (M estrado em Fitotecnia) - Universidade Federal de Viçosa, Viçosa.

12. Iamamoto, M.M.; A morelli Filho, S. Comportamento de fungicidas no controle da mancha de ramulária do algodoeiro (G ossypium hirsutum L.). In: V CONGRESSO BRASILEIRO DE ALGODÃ 0, 2005, Salvador. A nais. Campina Grande: EM BRA PA-CNPA, 2005. (CD-Room).

13. Kim, S.G.; Kim, K.W.; Park, E.W.; Choi, D. Silicon-induced cell wall fortification of rice leaves: a possible cellular mechanism of enhanced host resistance to blast. Phytopatology, v. 92, p. 10951103, 2002

14. Korndörfer, G.H.; Pereira, H.S.; Nolla, A. A nálise de silício: solo, planta e fertilizante. U berlândia: Universidade Federal de U berlândia, 2004 (Boletim Técnico). 24 p.

15. Machado, A.Q.; A ndrade, P.M.C.; Cassetari N eto, D. Controle químico de doenças da parte aérea do algodão em $M$ ato Grosso. In: II CONGRESSO BRASILEIRO DE ALGODÃO, 1999, Ribeirão Preto. Anais. Campina Grande: EMBRAPA-CNPA, 1999. p. 483-484.

16. M aranha, F.G.C.B.; Ramalho, M.A.P.; Farias, F.J.C. Estratégias de análise da reação de cultivares de algodoeiro a patógenos. Revista Brasileira Oleaginosas e Fibrosas, v. 6, n. 2, p. 565-575, 2002

17. M enzies, J., Bowen, P., Ehret, D., Glass, A. D .M. Foliar applica- tions of potassium silicate reduce severity of powdery mildew on cucumber, muskmelon, and zucchini squash. Journal of the A merican Society for Horticultural Science, v. 117, p. 902905, 1992.

18. M iyake, Y. \& Takahashi, E. Effects of silicon on the growth of cucumber plant in soil culture. Soil Science and Plant Nutrition, v. 29, p. 463-471, 1983.

19. Paiva, F.A. Doenças. In: EMBRAPA AGROPECUÁRIA DO OESTE (Eds). Algodão: tecnologia de produção. Embrapa Algodão, Dourados: Embrapa A gropecuária do oeste, 2001. p. 245-266.

20. Richetti, A.; Melo Filho, G.A. A spectos socioeconômicos do algodoeiro. In: EMBRAPA AGROPECUÁRIA DO OESTE (Eds). Algodão: tecnologia de produção. Embrapa Algodão, Dourados: Embrapa A gropecuária do oeste, 2001. p. 13-34.

21. Rocha, C.L.; Carvalho, C.L.; Oliveira, C.G. Avaliação de fungicidas no controle de ramulária (Ramularia aerola) na cultura do algodão. In: XXXVIII CONGRESSO BRASILEIRO DE FITOPATOLOGIA, 2005, Brasília. A nais. Brasília, 2005. p. 582 (Suplemento)

22. Rodrigues, F.Á; Benhamou, N.; Datnoff, L.E.; Jones, J.B.; Bélanger, R.R. Ultrastructural and cytochemical aspects of siliconmediated rice blast resistance. Phytopathology, v. 93, p. 535546, 2003.

23. Rodrigues, F.Á.; M cnally, D.J.; Datnoff, L.E.; Jones, J.B.; Labbé, C.; B enhamou, N.; M enzies, J.G.; Bélanger, R.R. Silicon enhances the accumulation of diterpenoid phytoalexins in rice: a potencial mechanism for blast resistance. Phytopathology, v. 94, p. 177183, 2004.

24. Shaner, G.; Finney, R.E. The effect of nitrogen fertilization on the expression of slow-mildewing resistance in $\mathrm{K}$ nox wheat. Phytopathology, v. 67, p. 1051-1056, 1977.

25. Zambolim, L.; Cruz Filho, J.; Vale, F.X.R.; Chaves, G.M. Emprego da calda Viçosa na cultura do tomateiro (Lycopersicum esculentum) para controle de doenças da parte aérea. Viçosa: UFV, 1990. 7 p. (Informe Técnico, 66). 